\title{
The challenge of predicting influenza
}

\author{
Julio A. Ramirez* \\ Division of Infectious Diseases, Department of Medicine, School of Medicine, University of Louisville, Louisville, KY, USA \\ *Correspondence: j.ramirez@louisville.edu
}

If we accept the idea that we are not able to "predict" the future of influenza, then we need to understand how we can prepare for the emergence of a new pandemic. Furthermore, if we cannot predict when our next problem is going to happen, we need to be able to recognize the problem as soon as it happens. This is why optimal influenza surveillance should be our first line of response. Another item of importance that is unpredictable is the virulence of a new, emergent strain of influenza. If the new strain is of low virulence, most patients will have a mild disease and some of them may even be asymptomatic. Most of the patients with this new virus will remain in the community setting (Figure 1, A1). Under the circumstances of a new pandemic with a low virulence virus, surveillance can be focused on visits to doctors' offices, drug stores, or illness reports from work or school. Local health departments typically perform these types of surveillance mechanisms. With the progression of the epidemic, and when abnormal hosts are infected, some patients will develop more serious disease requiring hospitalization (Figure 1, B1). On the other hand, if the novel virus is highly virulent, the initial cases will likely be complicated with pneumonia, and patients will require admission to the hospital, intensive care treatment, and mechanical ventilation (Figure 1, A2). In this type of scenario, the recognition of initial cases must occur in the hospital setting. As the pandemic progresses, patients with less severe disease will be recognized in the community (Figure 1, B2). A local outbreak of previously healthy patients with severe pneumonia requiring intensive care admission will be the first manifestations of a pandemic due to a highly virulent virus. Surveillance of severe pneumonia through

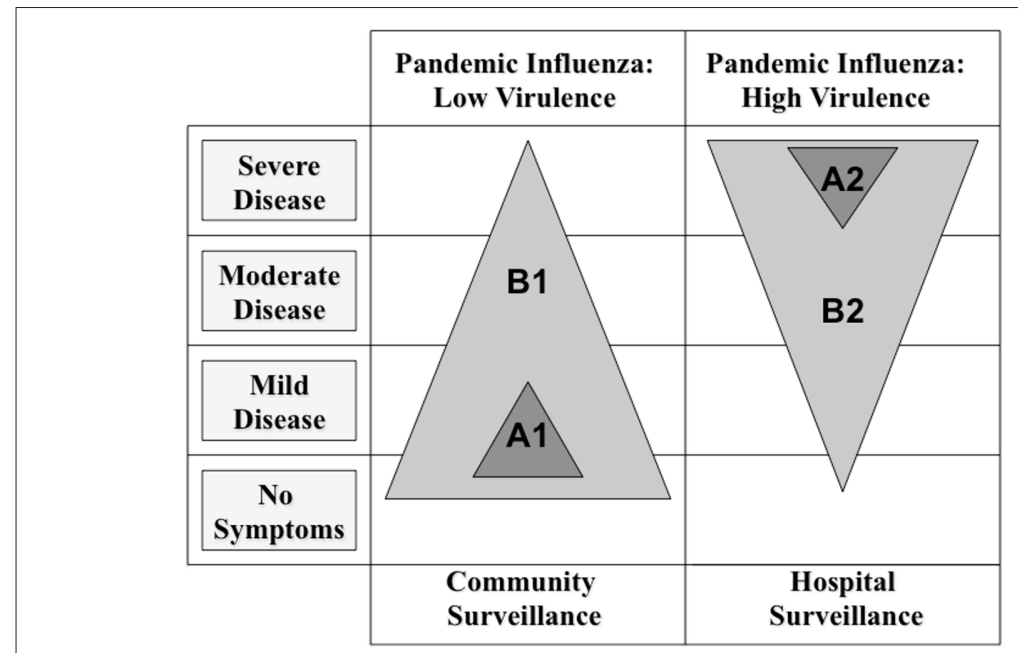

FIGURE 1 | Disease severity modeling and optimal surveillance approaches for emerging influenza viruses according to viral virulence.

a hospital infection prevention and control program will be our best approach for early identification of highly virulent virus.

Early recognition of a new pandemic should trigger a series of research studies. Investigators will need to characterize the new virus, define mechanisms of viral transmission, define clinical presentation, characterize patients at high risk for poor outcomes, and develop and test prevention and treatment strategies. To generate these types of knowledge in a short period of time requires a change in our standard approach of performing research. A traditional research model consists of investigators working in isolation in his or her laboratory. This must change to a new research model with basic and clinical investigators working in open collaboration. This new research model is defined by the United States National Institutes of Health as Clinical and Translational
Science. Collaboration of many experts in the field of influenza is happening in some Universities from around the globe. Well-funded, multidisciplinary collaboration is essential to face the challenges of influenza.

We may not be able to predict the influenza virus, but we can definitely study the virus and generate the necessary knowledge for its prevention and control.

Received: 27 February 2011; accepted: 28 February 2011; published online: 09 March 2011.

Citation: Ramirez JA (2011) The challenge of predicting influenza. Front. Microbio. 2:45. doi: 10.3389/ fmicb.2011.00045

This article was submitted to Frontiers in Cellular and Infection Microbiology, a specialty of Frontiers in Microbiology.

Copyright ( 2011 Ramirez. This is an open-access article subject to an exclusive license agreement between the authors and Frontiers Media SA, which permits unrestricted use, distribution, and reproduction in any medium, provided the original authors and source are credited. 